\title{
Perfil epidemiológico dos casos de sífilis congênita no estado de Minas Gerais no período de 2007 a 2017
}

\author{
Epidemiological profile of cases of congenital syphilis in the
} state of Minas Gerais from 2007 to 2017

\author{
F. T. Gomes; C. A. de Lima; P. L. S. Pires; S. V. de Oliveira*; T. Calegari \\ Universidade Federal de Uberlândia, 38408-100, Uberlândia - MG, Brasil. \\ *stefan@ufu.br
}

(Recebido em 28 de setembro de 2019; aceito em 13 de março de 2020)

\begin{abstract}
O objetivo é analisar os casos de sífilis congênita confirmados no estado de Minas Gerais (MG), no período de 2007 a 2017. Trata-se de um estudo descritivo, transversal e quantitativo realizado por meio da análise de informações secundárias disponibilizadas no Sistema de Informação de Agravos de Notificação. Foram avaliadas um total de 9.646 casos em que se identificou um acelerado aumento na frequência das notificações, passando de 1,8\% em 2007 para 25,3\% em 2017. Em relação à variável idade da criança, ocorreram 9.598 (99,5\%) dos casos na faixa etária menor de um ano. As características sociodemográficas maternas foram: $6.254(64,8 \%)$ estavam na faixa etária entre 20 a 34 anos, com predominância da raça parda em $4.768(49,4 \%)$ mulheres e para $1.552(16,0 \%)$ a escolaridade era de $5^{\circ}$ a $8^{\circ}$ série incompleta do ensino fundamental. O prénatal foi realizado por $85,5 \%$ das gestantes, em que $62,7 \%$ tiveram o diagnóstico de sífilis durante o pré-natal e $47,7 \%$ realizaram o tratamento inadequado. O esquema terapêutico prescrito para $29,4 \%$ (2.843) das crianças foi penicilina $\mathrm{G}$ cristalina. As características epidemiológicas dos casos de sífilis congênita são mulheres jovens, pardas, de baixa escolaridade, que realizam pré-natal, entretanto são diagnosticadas com a bactéria durante a gestação e não aderem ao tratamento adequado, transmitindo verticalmente a infecção para o concepto. As crianças diagnosticadas para a sífilis congênita estavam na faixa etária menor de um ano de idade. Medidas efetivas de diagnóstico, de adesão ao tratamento e educação em saúde são imprescindíveis para redução da prevalência alarmante desta Infecção Sexualmente Transmissível.
\end{abstract}

Palavras-chave: Sífilis Congênita; Saúde Pública; Cuidado Pré-natal.

The objective is to analyze the cases of congenital syphilis confirmed in the state of Minas Gerais (MG), between 2007 and 2017. Thus is a descriptive, cross-sectional and quantitative study performed through the analysis of secondary information provided in the Notification System. A total of 9,646 cases were identified in which an accelerated increase in the frequency of notifications was identified, from $1.8 \%$ in 2007 to $25.3 \%$ in 2017. Regarding the variable age of the child, 9,598 (99.5\%) of cases in the age group less than one year. The maternal sociodemographic characteristics were: 6,254 (64.8\%) were in the age group between 20 and 34 years old, with browns predominating in $4,768(49.4 \%)$ women and $1,552(16.0 \%)$ were schooling Incomplete 5 th to 8 th grade of elementary school. Prenatal care was performed by $85.5 \%$ of pregnant women, in which $62.7 \%$ had syphilis during prenatal care and $47.7 \%$ underwent inadequate treatment. The therapeutic regimen prescribed for $29.4 \%(2,843)$ of the children was crystalline penicillin G. Epidemiological characteristics of congenital syphilis are young, brown women with low schooling who are prenatal. However, they are diagnosed with the bacterium during pregnancy and do not adhere to the appropriate treatment, vertically transmitting the infection to the concept. Children diagnosed for congenital syphilis were under the age of one year. Effective measures of diagnosis, adherence to treatment and health education are essential to reduce the alarming prevalence of this Sexually Transmitted Infection.

Key words: Syphilis Congenital; Public Health; Prenatal Care.

\section{INTRODUÇÃO}

As Infecções Sexualmente Transmissíveis (IST) respondem por grande impacto na saúde global e, dentre elas, a sífilis é uma das mais comuns segundo a Organização Mundial da Saúde (OMS), estimando aproximadamente 6 milhões de novos casos a cada ano e é globalmente a segunda causa principal de morte fetal evitável, precedida apenas pela malária [1]. Causada pela bactéria Treponema pallidum, é uma doença de caráter sistêmico e exclusiva do ser humano, que quando disseminada por via transplacentária, ou seja, verticalmente da mãe para o concepto é denominado 
sífilis congênita (SC), podendo ser transmitida para o feto em qualquer fase da gestação, para o recém-nascido $(\mathrm{RN})$ durante o parto ou amamentação [2].

Considerada um agravo de notificação compulsória instituída por meio da Portaria n ${ }^{\circ} 542$ de 22 de dezembro de 1986, constitui SC casos de RN, natimorto ou aborto de mulher com sífilis não tratada ou tratada de forma inadequada. O tratamento adequado completo para estágio clínico da sífilis com penicilina benzatina deve ser iniciado em até 30 dias anterior ao parto. Uma vez que a infecção é por via placentária e pode ocasionar ao feto graves complicações, a SC é considerada um significativo problema de saúde pública mundial, apesar de apresentar testes diagnósticos sensíveis e tratamento efetivo de baixo custo [3].

Conforme o Boletim de Epidemiológico do estado de Minas Gerais (MG) de 2018, ao longo dos últimos cinco anos houve um acréscimo na taxa de incidência de casos com SC, passando de 2,4 casos/1000 nascidos vivos em 2013, para 7,0 casos/1000 nascidos vivos em 2017 [4]. Nesta perspectiva deve-se realizar captação de gestantes para as consultas de pré-natal e informá-las acerca dos exames diagnósticos existentes na atenção básica relevantes para o diagnóstico precoce de SC [4]. A associação da ocorrência da SC, na maioria das vezes, se deve ao fato de grávidas não realizarem a triagem para sífilis, e/ou aquelas que muitas vezes não são tratadas adequadamente ou sequer recebem tratamento [5].

O presente estudo teve como objetivo, analisar os casos de SC confirmados no estado de MG, no período de 2007 a 2017, notificados no Sistema de Informação de Agravos de Notificação (SINAN).

\section{MATERIAL E MÉTODOS}

Estudo descritivo, transversal e quantitativo executado por meio de informações secundárias disponibilizadas no banco de dados do SINAN. Foi solicitado por via eletrônica no site "Sistema eletrônico do serviço de informação ao cidadão" a base de dados das notificações dos casos de SC no período de 2007 até 2017, mediante o número de protocolo 25820.002510/2019-15 no dia 01/04/2019. A partir dos arquivos recebidos por e-mail, foram elencadas as variáveis disponibilizadas na ficha de notificação.

Foram incluídos todos os casos com SC notificados em MG, de acordo com a definição descrita na ficha de notificação da enfermidade, que constam na base de dados do SINAN entre o período de 2007 a 2017.

Em relação à notificação individual foram analisados a idade, sexo, raça/cor e o município de residência. No tocante aos antecedentes epidemiológicos da gestante/mãe, foram abordadas as variáveis: idade da mãe, raça/cor, escolaridade, se realizou pré-natal na gestação e quanto ao diagnóstico da sífilis materna: durante pré-natal, no momento do parto/curetagem, após o parto ou não realizado e ignorado.

Quanto aos dados laboratoriais da gestante/mãe foram considerados sobre a realização do teste não treponêmico e teste treponêmico no parto/curetagem, esquema de tratamento e se parceiros sexuais foram tratados concomitantemente a gestante. Pertinente aos dados laboratoriais da criança foi considerado a realização dos testes laboratoriais como: não treponêmico por sangue periférico, teste treponêmico após 18 meses, teste não treponêmico por líquor, titulação ascendente, evidência do Treponema pallidum. Em referência aos dados clínicos da criança, foi analisado: idade ao diagnóstico da SC, esquema de tratamento realizado e a evolução do caso.

As variáveis foram inseridas e analisadas pelos Softwares Excel (Microsoft) e Tabwin 3.32, averiguadas por estatística descritiva, representadas por números brutos, medidas de frequência relativa e absoluta e apresentados em forma de figuras e tabelas. A incidência (por 1000 nascidos vivos) foi calculada por ano da notificação, utilizando a informação do Sistema de Informações sobre Nascidos Vivos - SINASC para o estado de MG, disponibilizada pelo DATASUS.

A pesquisa foi realizada a partir de um banco de dados com informações secundárias, de domínio público, de livre acesso, no qual foram analisados dados não nominais dos pacientes, dispensando a submissão ao Comitê de Ética em Pesquisa (CEP) de acordo com a Resolução do Conselho Nacional de Saúde no 510, de 7 de abril de 2016 [6]. 


\section{RESULTADOS}

Foram registrados 9.646 casos de SC no estado de MG no período estudado. Houve um aumento na frequência dos casos notificados, passando de 1,8\% em 2007 para 25,3\% em 2017 conforme observado na Figura 1. A incidência média para o período analisado foi de 3,36 casos a cada 1000 nascidos vivos (variando de 0,69 no ano de 2007 a 9,36 por 1000 nascidos vivos no ano de 2017).

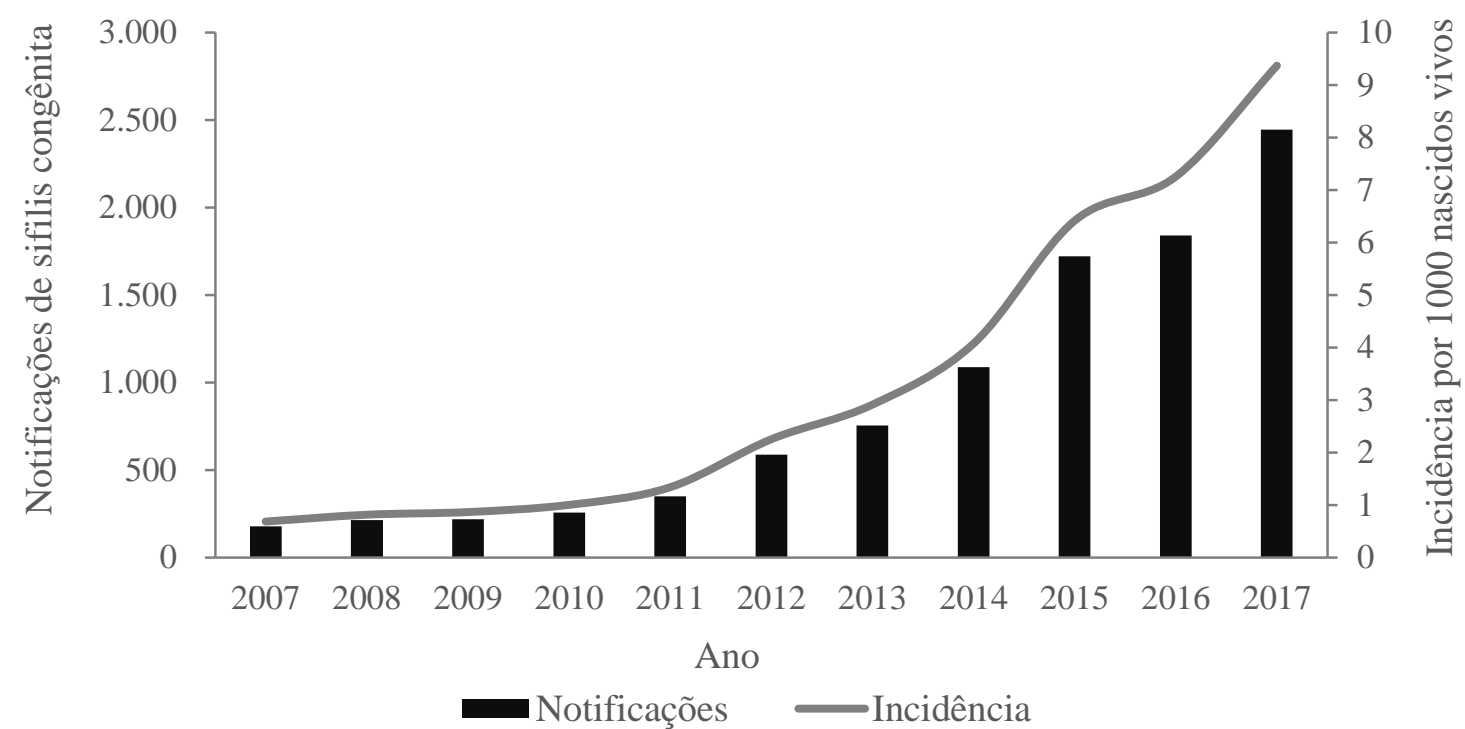

Figura 1 - Notificações e incidência dos casos de sífilis congênita no período de 2007 a 2017 registrados no Sistema de Informação de Agravos de Notificação em Minas Gerais.

Em relação à variável idade da criança na ocasião do diagnóstico, detalhada no campo notificação individual, observaram-se eventos desde menor de um ano até 12 anos. Na faixa etária menor de um ano ocorreram 9.598 (99,5\%) dos casos e as demais idades e variáveis gênero, raça/cor serão apresentadas na Tabela 1.

Tabela 1 - Número e frequência de casos notificados de sífilis congênita de acordo com a faixa etária da criança, gênero e raça/cor na ocasião da notificação em Minas Gerais.

\begin{tabular}{lcc}
\hline \multicolumn{1}{c}{ Variáveis } & Número de casos (n) & Frequência relativa (\%) \\
\cline { 1 - 1 } \multicolumn{1}{c}{ Faixa etária } & & \\
1 ano & 9.598 & $99,5 \%$ \\
$2-4$ anos & 25 & $0,2 \%$ \\
$5-7$ anos & 14 & $0,1 \%$ \\
$8-12$ anos & 3 & $0,03 \%$ \\
\multicolumn{1}{c}{ Gênero } & 6 & $0,06 \%$ \\
\cline { 1 - 1 } Feminino & & \\
Masculino & 4.657 & $48,2 \%$ \\
Ignorado & 4.491 & $46,5 \%$ \\
\multicolumn{1}{c}{ Raça/cor } & 498 & $5,1 \%$ \\
Parda & & \\
Branca & 4.131 & $42,8 \%$ \\
Preta & 1.933 & $20,0 \%$ \\
Amarela & 608 & $6,3 \%$ \\
Indígena & 33 & $0,3 \%$ \\
Ignorado & 6 & $0,06 \%$ \\
Total & 2.935 & $30,4 \%$ \\
\hline
\end{tabular}


A Unidade Federativa de MG tem 853 municípios, dos quais as notificações foram realizadas de acordo com o local de residência, como visualizado na Figura 2. As três cidades mais populosas do estado de MG e que representaram mais notificações de SC são: Belo Horizonte (2.375.151 habitantes), Uberlândia (604.013 habitantes) e Contagem (603.442 habitantes) [7].

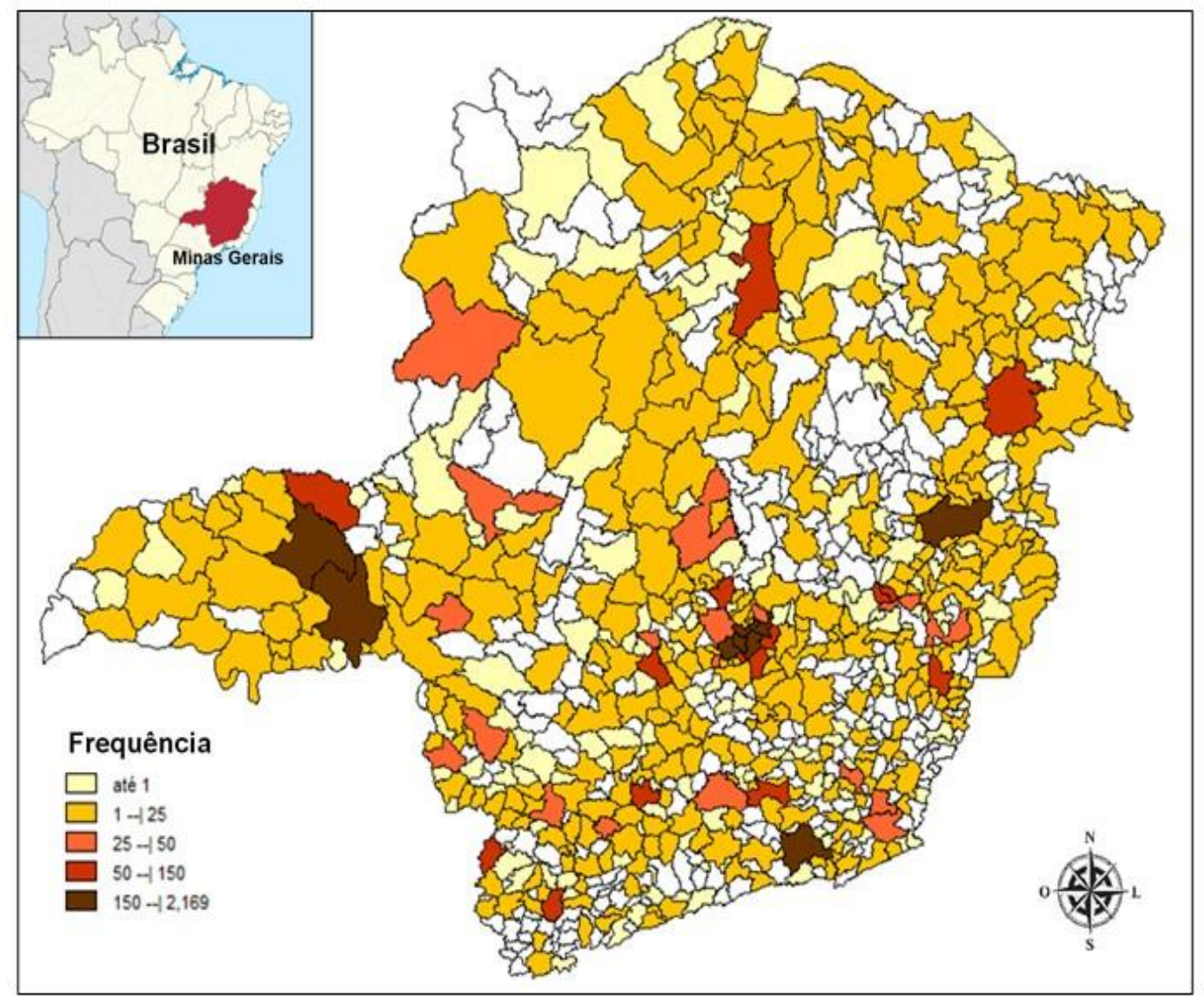

Figura 2 - Mapa da frequência dos casos notificados de sífilis congênita segundo o município de residência no estado de Minas Gerais nos anos de 2007 a 2017.

Em relação às características sociodemográficas quanto a faixa etária, raça, escolaridade, adesão ao pré-natal, momento do diagnóstico, esquema de tratamento e tratamento do parceiro pode-se visualizar o número de casos e frequência relativa conforme observado na Tabela 2.

Quanto aos testes laboratoriais da mãe durante o parto-curetagem, em 8.226 gestantes $(85,2 \%)$ o teste não treponêmico foi reagente, 665 não foram reagentes $(6,8 \%)$, em 391 gestantes ele não foi realizado $(5,2 \%)$ e $364(3,7 \%)$ foi ignorado. Em 4.110 pacientes $(42,6 \%)$ o teste treponêmico não foi realizado, $406(4,2 \%)$ foi não reativo, em $3.665(37,9 \%)$ gestantes ele foi reativo e $1.465(15,1 \%)$ casos foram ignorados ou em branco para essa variável nas fichas de notificação. No tocante aos dados laboratoriais da criança, as variáveis são apresentadas na Tabela 3. 
Tabela 2 - Predominância dos casos notificados de sífilis na gestação segundo características sociodemográficas maternas, diagnóstico segundo a assistência pré-natal, tratamento de gestante e do parceiro em Minas Gerais.

\begin{tabular}{|c|c|c|}
\hline Variáveis & $\begin{array}{c}\text { Número de casos } \\
\text { (n) }\end{array}$ & $\begin{array}{c}\text { Frequência relativa } \\
(\%)\end{array}$ \\
\hline \multicolumn{3}{|l|}{ Faixa etária } \\
\hline Ignorado & 197 & $2,0 \%$ \\
\hline $10-14$ anos & 86 & $0,8 \%$ \\
\hline $15-19$ anos & 2.258 & $23,4 \%$ \\
\hline $20-34$ anos & 6.254 & $64,8 \%$ \\
\hline $35-49$ anos & 850 & $8,8 \%$ \\
\hline $50-64$ anos & 1 & $0,01 \%$ \\
\hline \multicolumn{3}{|l|}{ Raça/cor } \\
\hline Parda & 4.768 & $49,6 \%$ \\
\hline Branca & 1.807 & $18,8 \%$ \\
\hline Preta & 1.358 & $14,0 \%$ \\
\hline Amarela & 55 & $0,5 \%$ \\
\hline Indígena & 12 & $0,1 \%$ \\
\hline Ignorado & 1.646 & $17,0 \%$ \\
\hline \multicolumn{3}{|l|}{ Escolaridade } \\
\hline Ignorado & 4.148 & $43,0 \%$ \\
\hline Analfabeto & 56 & $0,5 \%$ \\
\hline $1^{\circ}$ a $4^{\circ}$ série incompleta do ensino fundamental & 314 & $3,2 \%$ \\
\hline $4^{\circ}$ série completa do ensino fundamental & 237 & $2,6 \%$ \\
\hline $5^{\circ}$ a $8^{\circ}$ série incompleta do ensino fundamental & 1.552 & $16,0 \%$ \\
\hline Ensino fundamental completo & 982 & $10,1 \%$ \\
\hline Ensino médio incompleto & 943 & $9,7 \%$ \\
\hline Ensino médio completo & 1.196 & $12,6 \%$ \\
\hline Educação superior incompleta & 63 & $0,6 \%$ \\
\hline Educação superior completa & 72 & $0,7 \%$ \\
\hline \multicolumn{3}{|l|}{ Realizou pré-natal } \\
\hline Ignorado & 381 & $3,9 \%$ \\
\hline Sim & 8.255 & $85,5 \%$ \\
\hline Não & 1.010 & $10,6 \%$ \\
\hline \multicolumn{3}{|l|}{ Diagnóstico sífilis materna } \\
\hline Ignorado & 392 & $4,0 \%$ \\
\hline Durante o pré-natal & 6.051 & $62,7 \%$ \\
\hline No momento do parto-curetagem & 2.348 & $24,3 \%$ \\
\hline Após o parto & 787 & $8,16 \%$ \\
\hline Não realizado & 68 & $0,9 \%$ \\
\hline \multicolumn{3}{|l|}{ Esquema de tratamento } \\
\hline Inadequado & 4.604 & $47,7 \%$ \\
\hline Adequado & 2.000 & $20,7 \%$ \\
\hline Não realizou & 2.059 & $21,3 \%$ \\
\hline Ignorado & 983 & $10,3 \%$ \\
\hline \multicolumn{3}{|l|}{ Tratamento do parceiro } \\
\hline Ignorado & 2.026 & $21,0 \%$ \\
\hline Sim & 2.855 & $29,5 \%$ \\
\hline Não & 4.765 & $49,5 \%$ \\
\hline Total & 9.646 & $100 \%$ \\
\hline
\end{tabular}


Tabela 3 - Diagnóstico e tratamento de recém-nascidos notificados com sífilis congênita em Minas Gerais. Fonte: Sistema de Informação de Agravos de Notificação (2019).

\begin{tabular}{|c|c|c|}
\hline Variáveis & $\begin{array}{c}\text { Número de casos } \\
\text { (n) }\end{array}$ & $\begin{array}{c}\text { Frequência relativa } \\
(\%)\end{array}$ \\
\hline \multicolumn{3}{|l|}{ Teste não treponêmico - Sangue Periférico } \\
\hline Ignorado- Branco & 430 & $4,4 \%$ \\
\hline Reativo & 6.764 & $70,1 \%$ \\
\hline Não reativo & 1.521 & $15,7 \%$ \\
\hline Não realizado & 931 & $9,6 \%$ \\
\hline Total & 9.646 & $100 \%$ \\
\hline \multicolumn{3}{|l|}{ Teste Treponêmico - 18 meses } \\
\hline Ignorado- Branco & 6.575 & $68,1 \%$ \\
\hline Reativo & 34 & $0,3 \%$ \\
\hline Não reativo & 323 & $3,3 \%$ \\
\hline Não realizado & 2.714 & $28,1 \%$ \\
\hline Total & 9.646 & $100 \%$ \\
\hline \multicolumn{3}{|l|}{ Teste não treponêmico - líquor } \\
\hline Ignorado-Branco & 1170 & $12,1 \%$ \\
\hline Reativo & 272 & $2,8 \%$ \\
\hline Não reativo & 4.357 & $45,1 \%$ \\
\hline Não realizado & 3.847 & $39,8 \%$ \\
\hline \multicolumn{3}{|l|}{ Titulação ascendente } \\
\hline Ignorado-Branco & 2.854 & $29,5 \%$ \\
\hline Sim & 215 & $2,2 \%$ \\
\hline Não & 1.201 & $12,4 \%$ \\
\hline Não realizado & 5.376 & $55,7 \%$ \\
\hline \multicolumn{3}{|l|}{ Evidência de Treponema pallidum } \\
\hline Ignorado-Branco & 3.136 & $32,5 \%$ \\
\hline Sim & 199 & $2,0 \%$ \\
\hline Não & 1.649 & $17,0 \%$ \\
\hline Não realizado & 4.662 & $48,3 \%$ \\
\hline Total & 9.646 & $100 \%$ \\
\hline
\end{tabular}

O tratamento da criança com a penicilina G cristalina foi prescrita para $29,4 \%$ (2.843) das ocorrências; em 19,2\% (1.855) foi realizado a penicilina G procaína; 13,2\% (1.273) penicilina G benzatina; $15,9 \%$ (1540) receberam outros tratamentos, em 14,5\% (1.399) dos casos não houve esquema terapêutico e $7,6 \%$ (736) foram ignorados.

A evolução dos casos foi relata como: $8.379(86,8 \%)$ vivos, $182(1,9 \%)$ crianças apresentaram óbito por agravo notificado, $83(0,8 \%)$ por óbito de outra causa, $173(1,7 \%)$ abortos por SC, 352 $(3,6 \%)$ foram de natimortos e 477 (4,9\%) ignorado.

\section{DISCUSSÃO}

No período estudado de 2007 a 2017 em MG as notificações de SC corresponderam a 178 casos $(1,8 \%)$ em 2007 com aumento para 2.444 (25,3\%) em 2017. A frequência acumulada de SC encontrada neste estudo aumentou gradativamente ao decorrer desses 10 anos, de $0,92 \%$ para $11,5 \%$.

Na literatura científica não há estudos recentes de SC em MG sendo observado em Belo Horizonte, no período de 2001 a 2008, a incidência aumentada de 78,0\% no intervalo de tempo estudado: de 0,9 para 1,6 casos/1000 NV [8]. Ao longo dos últimos cinco anos notou-se o crescente aumento na taxa de notificação de SC em MG de acordo com o Boletim Epidemiológico Mineiro Sífilis (BEM), passando de 2,4 casos/1000 NV em 2013 para 7,0 casos/1000 NV em 2017 [4]. 
No Brasil em 2006 a taxa de incidência de SC foi de 2,0 casos/1.000 NV, houve um progressivo aumento a partir de 2010 de aproximadamente 2,2 casos e em 2016 observou-se um crescimento três vezes maior, passando para 6,8 casos/1.000 NV [9]. Comparando os últimos cinco anos analisados (2011 e 2016), nota-se que os aumentos mais expressivos apresentaram-se nos estados de Santa Catarina e Minas Gerais[9]. Dados nacionais sobre SC em menores de um ano de idade notificados no SINAN de 1998 a junho de 2017 foram de 159.890 casos, dos quais $70.558(44,1 \%)$ residiam na região sudeste [10].

Pesquisas recentes sobre as notificações de SC verificaram que a maioria dos casos foi de conceptos de mães jovens (entre 20 e 34 anos), com pouca escolaridade, diagnosticadas durante período gestacional e cujos parceiros sexuais não aderiram ao tratamento concomitante, fato que pode explicar a comorbidade que crianças menores de um ano venham a apresentar SC $[11,12]$.

Existe uma relação entre a infecção segundo escolaridade da mãe: quanto menor o período de estudo da mulher, maior a ocorrência de infecção pela sífilis e de SC, em que 60,6\% das participantes possuíam ensino fundamental incompleto [11]. Em relação à SC os aspectos maternos como a cor parda e a baixa escolaridade da gestante demonstram serem fatores de risco para aquisição da infecção. Estes dados designam a relevância das desigualdades socioeconômicas em desfechos graves e previsíveis como a infecção pelo Treponema pallidum, que podem ser facilmente evitáveis com medidas socioeducativas e melhorias na qualidade dos serviços de saúde [8].

Nesta investigação em MG 85,5\% das mulheres realizaram o pré-natal, demonstrando que a maioria frequentou essa modalidade de atendimento. Segundo o boletim epidemiológico de sífilis no ano de 2017, 81,8\% das mães de crianças com SC tiveram acesso ao pré-natal [9]. O que representa para a mulher um momento de grande valia no período gestacional, por permitir promoção da saúde, prevenção de possíveis intercorrências maternas e do concepto além de diagnóstico precoce e adequabilidade de tratamento [13].

Salienta-se que 62,7\% das gestantes do presente estudo de MG obtiveram a confirmação de infecção sifilítica durante o pré-natal. Investigação recentemente publicada evidenciou que a nível nacional, dos casos de SC notificados em $2015,78,4 \%$ das mães haviam realizado pré natal e destas, $51,4 \%$ tiveram o diagnóstico de sífilis durante a gravidez e 34,6\% no momento do parto/curetagem [14]. É durante a primeira consulta que as gestantes devem realizar o teste sorológico para sífilis e outras IST's a fim de detectar precocemente infecções [15].

Apesar da alta adesão das mulheres ao pré-natal na atual investigação, esses índices identificados no momento do parto em conciliação com um pré-natal de insucesso retratam uma assistência de qualidade inferior prestada à gestante e a desvalorização das medidas comprovadamente eficazes: o diagnóstico precoce e o tratamento adequado da sífilis na gestação [14]. O estudo realizado em 2016 retratou que mais de $90 \%$ das mulheres receberam assistência pré-natal, evidenciando a baixa qualidade deste cuidado para a identificação e tratamento das gestantes com o agravo [11]. Nesse sentido, a dificuldade de prevenção da transmissão vertical permanece no entrave do diagnóstico e do tratamento precoce e oportuno [15].

É preconizado pelo MS que toda gestante seja testada duas vezes para sífilis durante o pré-natal: no primeiro trimestre e no terceiro trimestre ( $28^{\mathrm{a}}$ semana) de gravidez. É obrigatória a realização imediatamente após a internação para o parto na maternidade de um teste treponêmico (ou teste rápido) e na disponibilidade do serviço o teste não treponêmico (ou VDRL). A Portaria do MS $\mathrm{n}^{\circ}$ 1.459 , de 24 de junho de 2011, com o objetivo de identificar precocemente as gestantes em risco de sífilis e garantir o tratamento precoce durante a gestação, instituiu o teste rápido no âmbito da Rede Cegonha como ações para prevenção e tratamento de IST [16].

No presente trabalho um percentual de $42,1 \%$ realizou o teste treponêmico e $92 \%$ das mulheres realizaram o teste não treponêmico durante o parto/curetagem, sendo a maioria reagente. $\mathrm{O}$ resultado da sorologia para sífilis no momento de sua realização possibilita o início imediato do tratamento com a primeira dose de penicilina benzatina [11]. Inferindo que nesta população o diagnóstico não é identificado como preconizado pelo MS, atrasando o diagnóstico e tratamento adequado.

A fim de ser considerada adequadamente tratada, conforme o Protocolo Clínico e Diretrizes Terapêuticas para Prevenção da Transmissão Vertical de HIV, Sífilis e Hepatites Virais (2018), a mulher deve ter esquema terapêutico com doses de penicilina benzatina ao estágio clínico da doença 
e finalizado pelo menos 30 dias antes do parto, com este manejo clínico documentado no cartão de pré-natal [10].

Mensalmente devem ser realizados nas gestantes os testes não treponêmicos optando sempre preferencialmente pelo mesmo teste para possível comparação dos resultados. Mesmo que a resposta ao tratamento ocorra de maneira adequada, o seguimento clínico deve continuar com o intuito de monitorar possível reativação ou reinfecção [10].

Em relação ao esquema de tratamento em mulheres diagnosticadas com sífilis neste estudo, apenas $20,7 \%$ obtiveram tratamento adequado e aproximadamente $50,0 \%$ realizaram tratamento inadequado. Resultados semelhantes foram encontrados no estudo realizado do Tocantins no qual $40,7 \%$ recebeu tratamento inadequado [17]. O mesmo ocorre quando a gestante não realiza o tratamento preconizado de maneira correta e observa-se um aumento da titulação em pelo menos duas diluições; persistência ou recorrência dos sinais e sintomas de sífilis ou ausência de queda esperada do título. Em caso de retratamento, recomenda-se a administração de 2,4 milhões de UI de penicilina benzatina por via intramuscular uma vez por semana durante três semanas e investigação de neurossífilis por meio de punção lombar [10].

Para suprimir os riscos de transmissão vertical da sífilis é essencial realizar adequadamente o tratamento da gestante e do parceiro sexual, pois a penicilina benzatina é considerada a única opção segura e eficaz por não causar toxidade ao feto e ter a capacidade de atravessar a barreira transplacentária, tratando mãe e feto ao mesmo tempo $[10,14]$.

O boletim epidemiológico de sífilis, publicado em 2018, desconsidera o tratamento do parceiro sexual da mãe como critério diagnóstico, atualizando a definição para confirmação dos casos de SC [3]. Entretanto, estudos nacionais contrapõem essa prerrogativa ao estabelecerem a profilaxia presuntiva do parceiro como fato primordial para evitar a reinfecção da gestante e de suma importância o fortalecimento da sua presença no serviço de saúde junto à parceira [8]. A principal causa do tratamento ineficaz da gestante está relacionada ao não tratamento do parceiro [15].

Quanto às características inerentes aos dados laboratoriais da criança, a maioria daquelas que realizaram o teste não treponêmico no presente estudo foram reativas. Pesquisa realizada no ano de 2017 foi observado que 51\% das crianças que realizaram o teste não treponêmico em sangue periférico foi reagente [17]. Em crianças menores de 18 meses, o resultado reagente só tem relevância quando a titulação encontrada for maior do que o título materno em pelo menos duas diluições, devendo ser confirmada com uma segunda amostra [10].

Para aqueles RN em que o resultado do VDRL não for reagente e persistir a suspeita de SC, deve-se repetir o teste com um mês, três, seis, 12 e 18 meses de idade, devido a possibilidade de ainda ocorrer a soroconversão. $\mathrm{O}$ seguimento só deve ser interrompido após dois exames não treponêmicos consecutivos não reagentes. Na dúvida ou impossibilidade de seguimento, o RN deve ser tratado [10].

Devido ao fato de grande parte das crianças com SC precoce serem assintomáticas ao nascer, o diagnóstico depende de alta suspeição clínica para a investigação da história materna, além de exame cuidadoso da criança exposta, pois o VDRL pode positivar com poucos meses após o nascimento [5]. Segundo o "Guia de referências técnicas e programáticas para as ações do plano de eliminação da SC", a infecção pela bactéria Treponema pallidum pode causar consequências graves ao concepto como sequelas motoras, cognitivas, neurológicas, visuais e auditivas [18].

É garantido o tratamento para todas as crianças cujas mães tiveram sífilis não tratada ou inadequadamente tratada o esquema terapêutico que deve ser iniciado o mais rápido possível, a fim de evitar as sequelas da doença ou minimizá-las [17, 19]. Ocorrendo alterações clínicas e/ou imunológicas, a penicilina $\mathrm{G}$ cristalina pode ser escolhida na dose de 50.000 UI/Quilograma $(\mathrm{kg})$ /dose, por via intravenosa, a cada 12 horas (nos primeiros 7 dias de vida) e a cada 8 horas (após 7 dias de vida), durante 10 dias ou penicilina $\mathrm{G}$ procaína $50.000 \mathrm{UI} / \mathrm{kg}$, dose única diária, intramuscular, durante 10 dias. A penicilina procaína poder ser considerada como droga de escolha em casos que a neurossífilis tenha sido afastada e o uso desta favorece a complementação do tratamento fora da unidade hospitalar. Em casos de presença de alteração liquórica, o esquema terapêutico deve ser a penicilina $\mathrm{G}$ cristalina [10].

No atual estudo a maior porcentagem das crianças notificadas evoluíram com vida e os demais desfechos foram: 3,6\% natimorto, 1,9\% com óbito por SC e 1,7\% casos de aborto. Evidencia-se um diagnóstico final com boa evolução das crianças notificadas com SC. 


\section{CONCLUSÃO}

As limitações para desenvolvimento desta pesquisa foram o uso de banco de dados secundários do SINAN que se condiciona à qualidade dos registros realizados pelos profissionais dos municípios brasileiros. Observou-se um número elevado de dados brancos ou ignorados, fato explicado por possível reflexo de situações em que o Sistema de Informação Saúde esteve inoperante ou ainda ausência do registro pelo profissional, o que resulta em informações indisponíveis.

Os resultados obtidos neste estudo possibilitaram conhecer as características epidemiológicas das notificações de SC no estado de MG, no qual na ocasião diagnóstica a faixa etária pediátrica mais acometida foi menor de um ano, o grupo de maior risco para o desenvolvimento de sífilis gestacional são mulheres em idade fértil de 20 a 34 anos, de baixa escolaridade e raça parda. A maioria das gestantes realizou o pré-natal e obtiveram o diagnóstico nesse período, entretanto as evidências são de tratamento inadequado. Esses fatores interferem de maneira racional na cadeia de transmissão da doença e explica o progressivo aumento da frequência do agravo nos anos estudados nesta, evidenciando que a SC ainda é um grave problema de saúde pública.

Evidencia-se a necessidade de mais estudos epidemiológicos para subsidiar possíveis ações de enfrentamento, contribuindo com a disponibilização de informações, tanto para profissionais de saúde, quanto para os gestores. Conclui-se a partir desse estudo a relevância de investir na saúde pública e organizar os serviços, ampliando o acesso à população para captar precocemente as gestantes, fixando-as ao acompanhamento de pré-natal com atenção integral qualificada. Nestes espaços de saúde deve-se orientar sobre a rotina mínima de exames preconizados pelo MS e garantir o tratamento oportuno e adequado da gestante.

\section{REFERÊNCIAS BIBLIOGRÁFICAS}

1. Korenromp EL, Rowley J, Alonso M, Mello MB, Saman Wijesooriya N, Guy Mahiané S, et al. Global burden of maternal and congenital syphilis and associated adverse birth outcomes-Estimates for 2016 and progress since 2012. PLoS One. 2019;14(2):1-17. doi:10.1371/journal.pone.0211720

2. Brasil $\mathrm{M}$ da $\mathrm{S}$. Agenda de ações estratégicas para redução da sífilis no Brasil. Ministério da Saúde [Internet]. 2017;34. Available from: http://www.aids.gov.br/pt-br/pub/2017/agenda-de-acoesestrategicas-para-reducao-da-sifilis-no-brasil

3. Secretaria de Vigilância em Saúde. Sífilis 2018. Bol Epidemiológico Sífilis Secr Vigilância em Saúde Ministério da Saúde. 2018;49(45):1-43.

4. Secretaria do Estado de Saúde de Minas Gerais. Boletim Epidemiológico Mineiro - sífilis. Secr Estado Saúde Minas Gerais. 2018;III.

5. Padovani C, De Oliveira RR, Pelloso SM. Syphilis in during pregnancy: Association of maternal and perinatal characteristics in a region of southern Brazil. Rev Lat Am Enfermagem. 2018;26. doi: 10.1590/1518-8345.2305.3019

6. Ministério da Saúde. Resolução No510, de 7 de abril de 2016. Conselho Nacional de Saúde. 2019;1-8.

7. Instituto Brasileiro de Geogradia e Esatística. Belo Horizonte: população residente. IBGE. 2019;99-100.

8. Lima MG, dos Santos RFR, Barbosa GJA, Ribeiro G de S. Incidência e fatores de risco para sífilis congênita em Belo Horizonte, Minas Gerais, 2001-2008. Cien Saude Coleiva. 2013;18(2):499-506.

9. Secretaria Estadual de Saúde. Sífilis 2017. Bol Epidemiológico [Internet]. 2017;48, n.36(2358-9450):41. Available from: http://www.aids.gov.br/pt-br/pub/2017/boletim-epidemiologico-de-sifilis-2017

10. Ministério da Saúde. Protocolo Clínico e Diretrizes Terapêuticas para Atenção Integral às Pessoas com Infecções Sexualmente Transmissíveis Ficha Catalográfica. Ministério da Saúde. 2015;120.

11. Domingues RMSM, Leal M do C. Incidence of congenital syphilis and factors associated with vertical transmission: Data from the birth in Brazil study. Cad Saude Publica. 2016;32(6):1-12. doi: 10.1590/0102-311X00082415

12. Soares LG, Zarpellon B, Soares LG, Baratieri T, Lentsck MH, Mazza V de A. Gestational and congenital syphilis: maternal, neonatal characteristics and outcome of cases. Rev Bras Saúde Matern Infant. 2017;17(4):781-9.

13. Kwon YS, Kim JS, Choi PP, Song JH, Dudina D. Caderno de Atenção Básica: Atenção ao Pré-Natal de Baixo Risco. Vol. 11, Journal of Industrial and Engineering Chemistry. 2005. 103-109 p.

14. Andrade ALMB, Magalhães PVVS, Moraes MM, Tresoldi AT, Pereira RM. Diagnóstico Tardio De Sífilis Congênita: Uma Realidade Na Atenção À Saúde Da Mulher E Da Criança No Brasil. Rev Paul Pediatr. 2018;36(3):376-81. doi: 10.1590/1984-0462/;2018;36;3;00011 
15. Lafetá KRG, Martelli Júnior H, Silveira MF, Paranaíba LMR. Sífilis materna e congênita, subnotificação e difícil controle. Rev Bras Epidemiol. 2016;19(1):63-74. doi: 10.1590/1980-5497201600010006

16. Ministério da Saúde. Portaria ${ }^{\circ} 1.459$, de 24 de Junho de 2011. Ministério da Saúde. 2019;1-9.

17. Cavalcante PA de M, Pereira RB de L, Castro JGD. Sífilis gestacional e congênita em Palmas, Tocantins, 2007-2014. Rev Sist Unico Saude Bras. 2017;26(2):255-64. doi: 10.5123/S1679-49742017000200003

18. Ministério da Saúde. Guia de Referências Técnicas e Programáticas para as ações do Plano de eliminação da Sífilis Congênita. 2010.

19. França ISX de, Batista JDL, Coura AS, Oliveira CF de, Araújo AKF, Sousa FS de. Factors associated to the notification of congenital syphilis: an indicator of quality of prenatal care. Rev Rede Enferm Nord. 2015;16(3):374-81. doi: 10.15253/2175-6783.2015000300010 